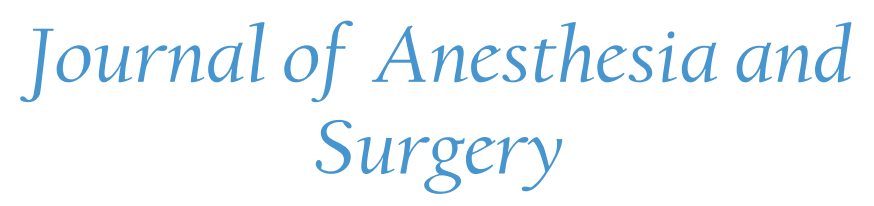

\title{
Minimizing Stress Response and Cognitive Dysfunction in Orthopedic Surgery: The Prospects of Epidural Anesthesia
}

\author{
Anna A. Ezhevskaya, MD, PhD ${ }^{1^{*}}$, Zhanna B. Prusakova, MD², Valery I. Zagrekov, MD, PhD ${ }^{3}$ \\ ${ }^{1}$ Leading Researcher of Anesthesiology and Intensive Care Department, Federal State Budgetary Institution "Privolzhsky Federal \\ Research Medical Centre" of the Ministry of Health of the Russian Federation \\ ${ }^{2}$ Senior researcher fellow of Anesthesiology and Intensive Care Department, Federal State Budgetary Institution "Privolzhsky \\ Federal Research Medical Centre" of the Ministry of Health of the Russian Federation \\ ${ }^{3}$ The chief of Anesthesiology and Intensive Care Department, Federal State Budgetary Institution "Privolzhsky Federal Research \\ Medical Centre" of the Ministry of Health of the Russian Federation
}

"Corresponding author: Anna A. Ezhevskaya, MD, PhD, Leading Researcher of Anesthesiology and Intensive Care Department, Federal State Budgetary Institution "Privolzhsky Federal Research Medical Centre" of the Ministry of Health of the Russian Federation, 603155, Nizhny Novgorod, Russia, Verkhne-Voljskaya naberejnaya, 18, E-mail: annaezh@gmail.com

\begin{abstract}
Purpose: To evaluate the effect of epidural anesthesia on immune and inflammatory changes, and to indicate their possible correlation with cognitive functions in patients undergoing orthopedic surgery.

Methods: Fifty-six female patients, aged 55-64 undergoing transpedicular fixations were randomly divided into two clinical groups: Group $1(\mathrm{n}=31)$ where epidural and general anesthesia were combined and Group $2(\mathrm{n}=25)$, in which general anesthesia with sevoflurane was administered. All the patients were assessed with the pain VAS at rest and during mobilization, and with the cognitive function standardized scales: MMSE, MoCA, and FAB. The plasma levels of cortisol, cytokines and were evaluated.

Results: Group 1 patients have demonstrated more adequate pain relief, lower cortisol and cytokines levels. In Group 2, there were $32 \%$ of patients with transient and mild cognitive dysfunction on postoperative day (POD) 1 as compared with Group $1(16 \%)$. All the cognitive impairments were gone by POD 5 after the surgery.

Conclusion: Epidural anesthesia (EA) has proved to be the most adequate analgesia and yielded the highest total scores in the cognitive tests on POD 1, as compared with general sevoflurane anesthesia and postoperative opioid analgesia. EA can modulate adaptive immunity improving the distribution of white blood cells. However, further research is required to obtain more results regarding the pathogenesis of POCD in patients after spinal surgery.
\end{abstract}

Received date: September 8, 2017

Accepted date: October 4, 2017

Published date: October 5, 2017

Citation: Ezhevskaya, A.A., et al. Minimizing Stress Response and Cognitive Dysfunction in Orthopedic Surgery: The Prospects of Epidural Anesthesia. (2017) J Anesth Surg 4(2): 100- 107.

DOI: $10.15436 / 2377-1364.17 .089$

Keywords: Epidural anaesthesia; Cognitive dysfunction; Stress response; Orthopedic surgery

\section{Introduction}

The current technologies of perioperative anesthesia and analgesia in orthopedics and spine surgery effectively address the issues of pain relief, being aimed to prevent possible complications and side effects in the postoperative period. Major orthopedic procedures, including spine surgery, are usually accompanied by massive tissue damage, severe blood loss, surgical stress response, and postoperative pain, which can cause immune suppression by itself ${ }^{[1-3]}$. The surgical stress response begins with initial changes in the neuroendocrine balance, and it can also result from alterations in homeostasis. Postoperative cognitive dysfunction (POCD) is a major consequence of this. It is essentially "deterioration in cognition temporally associated with surgery" $[4,5]$, and might lead to a higher mortality rate within three post-operative months ${ }^{[6]}$. A number of studies have revealed that the inflammatory response is a key factor in the development of postoperative cognitive dysfunction (POCD) $)^{[7-10]}$. 
Deterioration in the endocrine response to surgery can heighten the risk of developing POCD. Higher cortisol levels postoperatively have also been shown to potentially result in cognitive disorders ${ }^{[11]}$. Preclinical trials also suggest that inflammation is a possible pathogenic mechanism for the development of $\operatorname{POCD}^{[7,10]}$.

High levels of inflammatory markers have been associated with postoperative cognitive impairment ${ }^{[12]}$. It is considered that an excessive level of stress markers may indicate a greater risk of developing POCD, as a side event associated with the application of general anesthesia ${ }^{[13,14]}$. Furthermore, an increased level of such markers as proinflammatory cytokines may account for the surgical stress-induced immune response, which manifests itself within the hippocampus postoperatively and has been shown to cause POCD in murine models. The cytokines in question include interleukins (IL-6, IL-1b ${ }^{[12-14]}$ and tumor necrosis factor-alpha $(\mathrm{TNF})^{[15]}$.

Heterogeneous data are found in studies looking into the effect of regional anesthesia (RA) as compared to that of general anesthesia (GA) with regard to POCD rates. For instance, Rasmussen et al. ${ }^{[16]}$ reveal no significant difference between RA and GA in the rates of POCD; however, another study demonstrates a significantly poorer cognitive function in patients undergoing GA rather than $\mathrm{RA}^{[17]}$. As previously discussed, RA attenuates the stress response to a greater degree than GA. This suggests that although the levels of surgical stress can be associated with POCD, its role as a key factor is still uncertain ${ }^{[18]}$.

The medical and social significance of the issue is due to an increased rate of complications and, therefore, higher delayed mortality, longer duration of hospital stay and rising treatment $\operatorname{costs}^{[4]}$. Currently, the risk factors of POCD have been accurately identified with the aid of evidence-based medicine. The factors include the severity, duration and type of surgical procedures, elderly age, previous chronic pain, and patients' education level, along with anesthesia awareness episodes or critical hypotension in the past ${ }^{[5,19]}$.

At the same time, there are no conclusive data on the effect of anesthesia techniques on the development of POCD, and there is still little research into the role of regional anesthesia in POCD prevention. It should be noted that the majority of studies have investigated the risk factors of POCD in aged patients as the most vulnerable group in terms of postoperative delirium and POCD ${ }^{[19]}$. However, working-age patients are exposed to no less danger associated with traumatic surgery, the risk of cognitive disturbances and social or psychological disadaptation, along with delayed postoperative recovery, discharge and return to normal life and social functions ${ }^{[20]}$. For these reasons, anesthesiologists should be constantly looking for new approaches for perioperative anesthesia and analgesia, to reduce the inflammatory stress response, and the possible development of cognitive dysfunction.

We have hypothesized that spine surgery may contribute to the development of systemic inflammation and hence the inflammation of the central nervous system, which might lead to an increase in the inflammatory and immune markers, accompanied by cognitive disturbances.

The purpose of the study was to evaluate the effect of epidural anesthesia on immune and inflammatory changes, and to indicate their possible correlation with cognitive functions in patients undergoing orthopedic surgery.

\section{Methods}

This prospective randomized study has been approved by the Institutional local ethics committee, and its all participants have provided their written informed consent. The study included 56 female patients aged 55 - 64, with the following degenerative diseases: lumbar osteochondrosis with multilevel spinal stenosis. All the patients underwent orthopedic lumbar surgery such as transpedicular fixation of two or three levels, with spinal decompression, over the period from October 2015 to May 2017.

Excluded from the study were patients unable to understand the study protocol or to handle the infusion pump/ catheter system, or with any known contraindications to the medications under study or to regional anesthesia. Further excluded were patients suffering from insulin-dependent diabetes mellitus, neuropathy in low extremities of any etiology, from demyelinating diseases, and from hepatic or renal failure; patients with any additional surgical site outside the back intended for catheter placement; patients using opioids chronically or actively abusing illicit substances; pregnant patients or those unable to communicate with the researchers and hospital staff due to deafness or blurred vision. Moreover, the exclusion criteria were extended to ischemic stroke within the past 12 months, the syndrome of cognitive impairment and dementia under 23 scores by the Mini-Mental State Examination (MMSE); to persistent neurological deficit resulting from stroke; to clinically significant anxiety and/or depression according to the Hospital Anxiety and Depression Scale ( $\geq 11$ points); physical status classification system by the American Society of Anesthesiologists (ASA) $>3$; to the patient's educational level being under nine grades; to mental illnesses, and to the duration of anesthesia over three hours.

\section{Study Protocol}

All the patients were randomly assigned to one of the two treatment groups - Group $1(\mathrm{n}=31)$ where epidural and general anesthesia were combined, and Group $2(\mathrm{n}=25)$, in which general anesthesia was administered. The randomization was effected with the aid of sealed envelopes containing group assignments based on a computer-generated randomization sequence (http://www.randomizer.org). Both groups were comparable in gender, anthropometric features and perioperative blood loss. In Group 1, patients were administered combined anesthesia - epidural anesthesia with fentanyl and ropivacaine, and endotracheal anesthesia with sevoflurane. All procedures were performed by an attending regional anesthesiologist experienced in both regional and general anesthesia techniques, and by the same surgical team. To test and evaluate the presence and severity of cognitive dysfunction, a neurologist expert in determining the cognitive status was involved.

\section{Epidural anesthesia}

After the insertion and positioning of a peripheral intravenous (IV) catheter, standard noninvasive monitors were applied, and oxygen was given via a face mask or nasal cannulas. Patients received IV midazolam and fentanyl titrated to their comfort but allowing them to maintain verbal responsiveness. The procedural area designated was cleaned with chlorhexidine, and the patients were covered with a clear fenestrated sterile 
drape. Following the local infiltration of $5 \mathrm{ml}$ of $2 \%$ lidocaine, the patients underwent puncturing and the insertion of the epidural catheter in the prone position 3 - 4 interspinous spaces above the access planned, the catheter being positioned $4-5$ $\mathrm{cm}$ cranially before the surgery. The catheter was secured with an aseptic sticker. After performing an aspiration test and giving the test dose ( $2 \mathrm{ml}$ solution of $2 \%$ lidocaine), a bolus of $3-7$ $\mathrm{ml}$ of $0.5 \%$ ropivacaine with $100 \mathrm{mg}$ fentanyl was administered via the catheter. Throughout the surgical procedure, a mixture of $0.2 \%$ ropivacaine with fentanyl $(2 \mathrm{mg} / \mathrm{ml})$ was being infused epidurally at a rate $2-8 \mathrm{ml}$ per hour.

During the 48 hours of the early postoperative period, patients in Group 1 were getting epidural analgesia with the same mixture of $0.2 \%$ ropivacaine and fentanyl at a rate of $2-8$ $\mathrm{ml}$ per hour, disposable elastomeric infusion pumps being applied. In Group 1 patients, the epidural catheter was removed on the second postoperative day (POD 2).

\section{General anesthesia}

The induction of anesthesia in both groups was performed with IV propofol $(2 \mathrm{mg} / \mathrm{kg})$ and fentanyl $(2 \mathrm{mg} / \mathrm{kg})$. Prior to intubation, rocuronium was used to ensure a neuromuscular blockade at a dose of $0.6 \mathrm{mg} / \mathrm{kg}$. Before the section and the most traumatic stages of surgery, patients in Group 2 underwent general anesthesia with sevoflurane (1.2 - 1.7 MAC); they were infused fentanil at a rate of $0.002-0.003 \mathrm{mg} / \mathrm{kg}$ per hour, and administered a bolus dose of $50-100 \mathrm{mcg}$. Postoperative pain management in Group 2 was performed by administering IV morphine via the Patient-Controlled Analgesia (PCA) infusion system. In Group 1 patients, general anesthesia was achieved by the inhalation of sevoflurane $(0.7-0.8 \mathrm{MAC})$ with an assessment of the anesthesia depth according to a Bispectral Index (BIS) monitor (Covidian, Mansfield, MA) within a target range of $50-60 \%$ in both groups. All the patients received standard perioperative monitoring. The visual analogue scale (VAS) was utilized to evaluate the intensity of postoperative pain at rest and during mobilization. Pain assessment ranged from " 0 " (no pain) to " 10 " (worst pain imaginable). At the conclusion of surgery, all the patients were extubated and transferred to the recovery room and then to their ward. Following the extubation, they were evaluated neurologically to detect potential deficits.

\section{Immune and cytokine testing}

Serum was sampled at the following time points: 1 before surgery; 2 - at the time of the surgical incision; 3 - at the most traumatic point of the surgery; 4 - at the end of surgery; 5 - on POD 1;6 - on POD 3; 7 - on POD 5. At each point, whole blood was obtained by IV puncture and collected with EDTA. A 2-ml blood sample was used for blood cell count and the remainder was centrifuged to obtain plasma, which was frozen to $-112^{\circ} \mathrm{F}$ for further analysis. The plasma concentrations of epinephrine and cortisol were measured using an immunosorbent assay kit (CORTISOL, Diagnostics Biochem Canada Inc., Canada, ON), with a sensitivity limit of $0.4 \mu \mathrm{g} / \mathrm{dl}$. C-reactive protein was measured at four time points $(1,4,6$, and 7$)$ with the use of immunoassay kits (ELISA, USA). The plasma levels of interleukins (IL-1 $\beta$, IL-6, IL-8, and IL-10) were measured at time points 1, 4, 5, and 6 with enzyme-linked immunosorbent assay (ELISA) kits, the detection levels being as follows: 0.125 $\mathrm{pg} / \mathrm{ml}$ for human IL- $1 \beta, 0.15 \mathrm{pg} / \mathrm{ml}$ for IL-6 and IL-8 and $0.5 \mathrm{pg} /$ $\mathrm{ml}$ for IL-10.

The membrane markers for phenotyping blood cells (the expression of immune cell surface receptors - the cluster of differentiation: HLA-DR+/CD3-, HLA-DR+/CD3+, HLA-DR, CD3, CD4, CD8, CD16, CD19 CD16/56+ и CD16/56+/CD3+) were measured before surgery, after surgery, on POD 1 and POD 3 , using monoclonal antibodies kits according to the cytofluorometry technique (Navios, Beckman Coulter Inc., USA).

\section{Cognitive testing}

To assess the severity of postoperative cognitive dysfunction, we resorted to the standardized questionnaires contained in the Montreal cognitive assessment scale (MoCA). To test frontal dysfunction, the frontal assessment battery (FAB) was employed. All the participants underwent cognitive analysis according to the quick assessment scales of MoCA and MMSE (mini-mental state examination test), despite the low sensitivity of the latter. The MoCA test battery serves to quickly assess mild cognitive dysfunction. This test evaluates memory, frontal functions (visual-constructive skills, the ability to speak fluently and to think abstractly, etc.), the nominative function of speech and visual-dimensional praxis. Alternative variants of assignments were chosen randomly and used before and after the surgery to increase the sensitivity of the tests. Reduction by one standard deviation from the point scored on the MoCA scale was considered to be a POCD criterion in the postoperative period as compared with the preoperative testing ( $\mathrm{Z}$ score $\geq 1.0$ ).

The Frontal Assessment Battery (FAB) was used to assess the frontal dysfunction, the goal being to make a quick screening for dementia, which mainly affects the frontal lobes and subcortical cerebral structures, along with the speech functions - conceptualization, vocabulary, and praxis including simple and complicated choice reactions. FAB assesses the grasping reflex, indicating a failure of the frontal lobe in adults. The results of FAB testing depend on the total number of points scored: $16-18$ points correspond to the normal frontal function, 12 - 15 - to moderate impairment, and 12 points - to severe frontal dysfunction. The duration of a patient's testing is $10-20$ minutes. The alternative variants of assignments chosen randomly were used before and after the surgery to increase the sensitivity of the tests. Reduction by one standard deviation from the point scored in the FAB testing was regarded as a POCD criterion in the postoperative period comparing with the preoperative testing ( $\mathrm{Z}$ score $\geq 1.0$ ). All the assessments of cognitive functions were made at the $1^{\text {st }}$ stage (the initial stage before surgery), the $5^{\text {th }}$ stage - on POD 1 , the $7^{\text {th }}$ stage - on POD 5 , and the $8^{\text {th }}$ stage - on POD 7.

\section{Statistical Analysis}

Statistical analysis was conducted according to the type of data distribution and the conditions of criterion applicability with the aid of STATISTICA software package (version 10.0, Stat Soft Inc.). We have employed non-parametric statistical methods and linear regression.

Values were reported as the mean and the standard deviation for parametric data, or the median with a $25-75 \%$ interquartile range for nonparametric data. Parametric variables were checked for normality using the Shapiro-Wilk test. The Mann-Whitney U statistical test was applied to nonparametric and ordinal variables. Comparison of the two groups was per- 
formed using the Student $\mathrm{t}$ test (for normally distributed data) and the Wilcoxon signed-rank test. Differences were considered statistically significant at $\mathrm{P}<0.05$.

\section{Results}

All the patients have completed the study without early elimination. Both groups were comparable in their demographic and clinical parameters (gender, weight, height, ASA, the type and duration of the surgery, perioperative blood loss) (Table 1).

Table 1: Clinical and Demographic Data (Mean \pm Standard Deviation).

\begin{tabular}{|l|c|c|}
\hline Parameter & Group 1 (n= 31) & Group 2 (n= 25) \\
\hline Level of Education & $10.1 \pm 0.9$ & $9.8 \pm 1.1$ \\
\hline Age (yrs)* & $57.3 \pm 3.6$ & $58.6 \pm 2.5$ \\
\hline Length of surgery, min & $148.3 \pm 12.7$ & $150.2 \pm 13.7$ \\
\hline $\begin{array}{l}\text { Blood loss during surgery, } \\
\text { ml }\end{array}$ & $350.6 \pm 30.5$ & $330.7 \pm 40.2$ \\
\hline $\begin{array}{l}\text { BIS at traumatic time } \\
\text { point, \% }\end{array}$ & $51.6 \pm 2.5$ & $52.2 \pm 1.9$ \\
\hline $\begin{array}{l}\text { Mean blood pressure at } \\
\text { traumatic time point, mm } \\
\text { Hg }\end{array}$ & $68.5 \pm 6.3^{*}$ & $85.7 \pm 4.7$ \\
\hline
\end{tabular}

$* P<0.05$ for comparison between groups, Values are mean \pm standard deviation.

Hemodynamic monitoring has shown that the use of epidural analgesia does not lead to life-threatening cardiovascular disorders, despite the fact that Group 1 has demonstrated a mean blood pressure which was significantly lower than in Group 2 from the statistical standpoint. However, in both groups there were no episodes of significant or long reduction in either the systolic or mean blood pressure, which could seriously affect the results of the neuropsychological assessment. Furthermore, we have not detected a critical decrease in the mean blood pressure, which would require the administration of a vasopressor. In both groups we have observed a comparable level of the BIS-index and its unidirectional changes during the surgical stages. It has been revealed that the BIS-index was within the range of 50$55 \%$ during the most traumatic stages of surgery. It should also be noted that in both groups there were no awareness episodes, either spontaneous or induced, during the anesthesia, despite the fact that they significantly differed by MAC of Sevoflurane.

\section{Postoperative pain}

The comparative analysis of postoperative pain syndrome has shown that Group 1 patients suffered pain of significantly less intensity, both at rest and during mobilization. The corresponding self-rated pain levels are shown in Table 2. Additional administration of IV morphine in Group 1 was not virtually needed. Patients in Group 1 used less IV morphine than the patients in Group 2 at all time points, with statistical significance achieved for POD 1 and POD 2. The patients in Group 2 complained of moderate and sometimes severe pain, the daily morphine requirements being $22.3 \pm 4.3 \mathrm{mg}$.
Table 2: Evaluation of Postoperative Pain at rest and during mobilization.

\begin{tabular}{|c|l|l|l|l|}
\hline \multirow{2}{*}{$\begin{array}{l}\text { Time } \\
\text { points } \\
\text { (hours) }\end{array}$} & \multicolumn{2}{l|}{ Group 1 $(\mathbf{n}=\mathbf{3 1})$} & \multicolumn{2}{l|}{ Group 2 $(\mathbf{n}=\mathbf{2 5})$} \\
\cline { 2 - 5 } & at rest & mobilization & at rest & mobilization \\
\hline $\mathbf{0}$ & $0.8 \pm 0,1^{*}$ & $2.1 \pm 0.7$ & $2.4 \pm 0.7$ & $2.9 \pm 0.8$ \\
\hline $\mathbf{2}$ & $1.2 \pm 0.3^{*}$ & $3.1 \pm 1.3^{*}$ & $3.0 \pm 1.4^{*}$ & $6.7 \pm 0.7$ \\
\hline $\mathbf{3}$ & $1.7 \pm 0.2^{*}$ & $2.7 \pm 1.0^{*}$ & $2.9 \pm 1.3^{*}$ & $5.6 \pm 0.6$ \\
\hline $\mathbf{6}$ & $1.5 \pm 0.2^{*}$ & $2.6 \pm 0.6^{*}$ & $2.9 \pm 1.2^{*}$ & $4.8 \pm 1.1$ \\
\hline $\mathbf{1 2}$ & $2.0 \pm 0.3^{*}$ & $2.5 \pm 0.6^{*}$ & $2.5 \pm 1.6^{*}$ & $4.1 \pm 1.2$ \\
\hline $\mathbf{1 6}$ & $1.8 \pm 0.4^{*}$ & $2.8 \pm 0.9^{*}$ & $2.9 \pm 1.5^{*}$ & $4.9 \pm 0.2$ \\
\hline $\mathbf{2 4}$ & $2.5 \pm 0.5^{*}$ & $3.6 \pm 0.8$ & $3.5 \pm 1.7$ & $5.3 \pm 0.7$ \\
\hline $\mathbf{3 6}$ & $2.7 \pm 0.6^{*}$ & $2.8 \pm 0.4^{*}$ & $2.7 \pm 0.5^{*}$ & $4.8 \pm 0.5$ \\
\hline $\mathbf{4 8}$ & $1.8 \pm 0.2^{*}$ & $2.5 \pm 0.3^{*}$ & $2.5 \pm 0.8^{*}$ & $4.5 \pm 0.9$ \\
\hline
\end{tabular}

* $P<0.05$ for comparison between groups, Values are mean \pm standard deviation.

\section{Assessment of stress and immune response}

The study has shown that the plasma levels of the main inflammatory cytokines IL-6, IL-1 $\beta$, IL-8, IL-10, C-reactive protein, and cortisol were significantly higher in patients of Group 2 as compared with Group 1, at points 3, 4, 5, $6(P<$ 0.05 for all). At the same time, we have observed unidirectional changes of stress markers in both groups (Figure 1). In Group 1 patients, cortisol levels were highest at the traumatic period of the surgery, returning to normal values by POD 1 . The plasma levels of IL- 6 were on the increase at the end of surgery, with the maximum value observed on POD 1 and falling insignificantly towards POD 3 in both groups $(P<0.05)$ (Figure 1). They had completely normalized by POD 7 in both groups.

The assessment of the inflammatory response has demonstrated a severe inflammatory reaction in $79 \%$ of patients in Group 2, such as leukocytosis (WBC up to $19.1 \pm 3.5 \times 10 * 9 / 1$ ) and an increase in the acute-phase protein levels (fibrinogen up to $9.1 \pm 2.7 \mathrm{~g} / \mathrm{l}$, and C-reactive protein to $180.6 \pm 25.4 \mathrm{mg} / \mathrm{l}$ ). This occurred in combination with dysproteinemia, according to the erythrocyte sedimentation rate measurements.

The study has revealed unidirectional significant changes in natural killer cells (NK-cells, CD16/56+) in both groups, their number increasing towards the end of surgery, and this trend continuing until POD 3. Nevertheless, Group 2 revealed a significantly greater number of NK-cells at the end of surgery, on POD 1 and POD $3(P<0.05$ for all) as compared with Group 1. At the same time, the CD3-surface marker was significantly reduced in both groups on POD 1 and POD 3, which testifies to immune depression. The marker was considerably lower in Group 2 than in Group 1. 


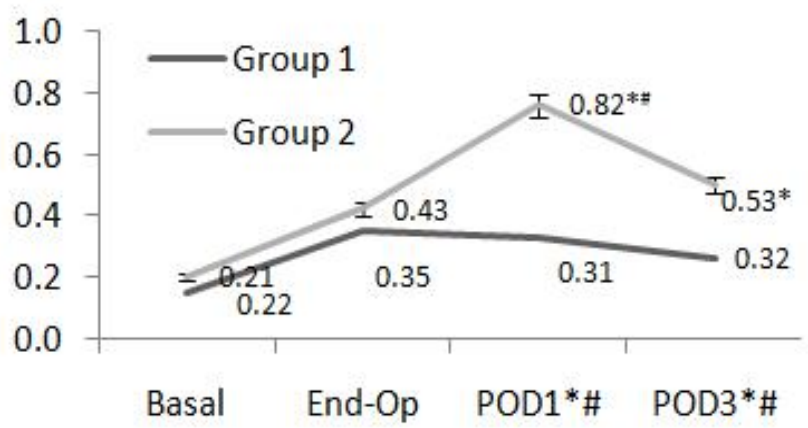

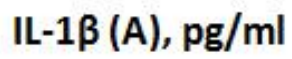

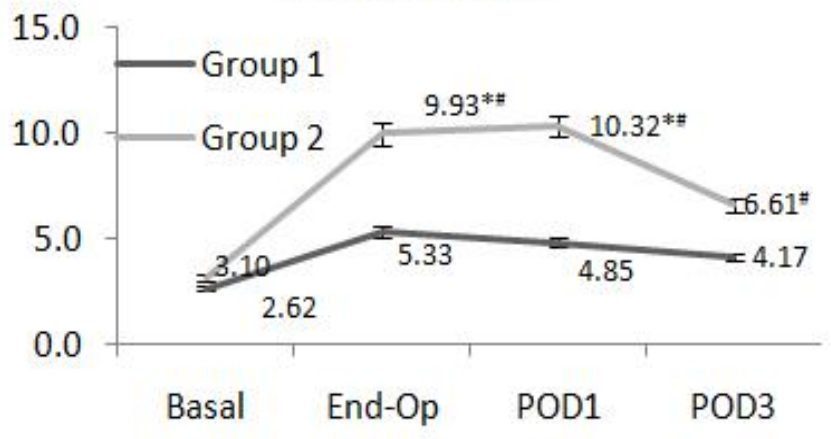

IL-10 (C), $\mathrm{pg} / \mathrm{ml}$
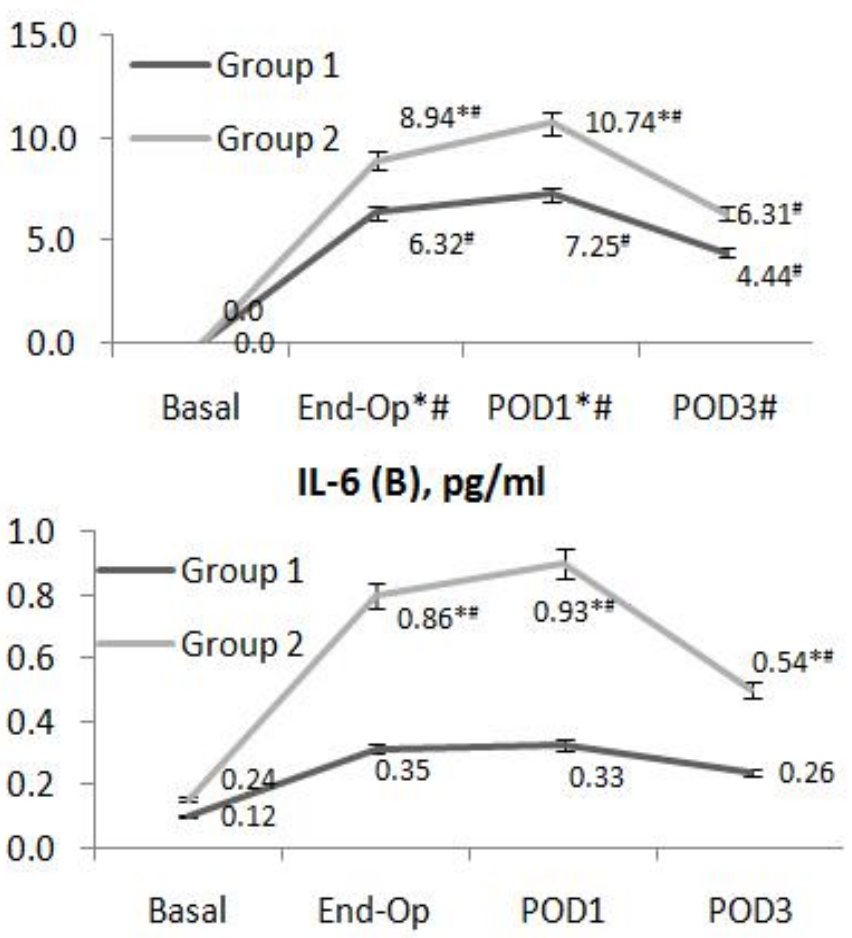

IL-8 (D), pg/ml

Figure 1: Assessment of the plasma levels of cytokines IL-1 $\beta$, IL-6, IL-8 IL-10 at Points $1,4^{*}, 5^{*}$, and $6^{*}(* P<0.05$ for comparison between groups, \# $P<0.05$ for comparison with the baseline).

The ratio of CD-4 (T-helpers) and CD-8 (cytotoxic cells) surface markers has been the complex criterion of the immune status. This parameter has undergone opposite changes in the two groups under study. Group 1 patients have indicated a significantly increased CD4/CD8 ratio and a much higher number of B-cells (B-lymphocytes), both parameters to normalize by POD 3. At the same time, the CD4/CD8 ratio in Group 2 was characterized by a severe and significant decline, particularly on POD 1 ( $P<0.05$, Figure 2). Significant changes in cortisol, C-reactive protein and IL-6 have had strong positive correlations among themselves and with the self-rated pain at the end of surgery and on POD 1.

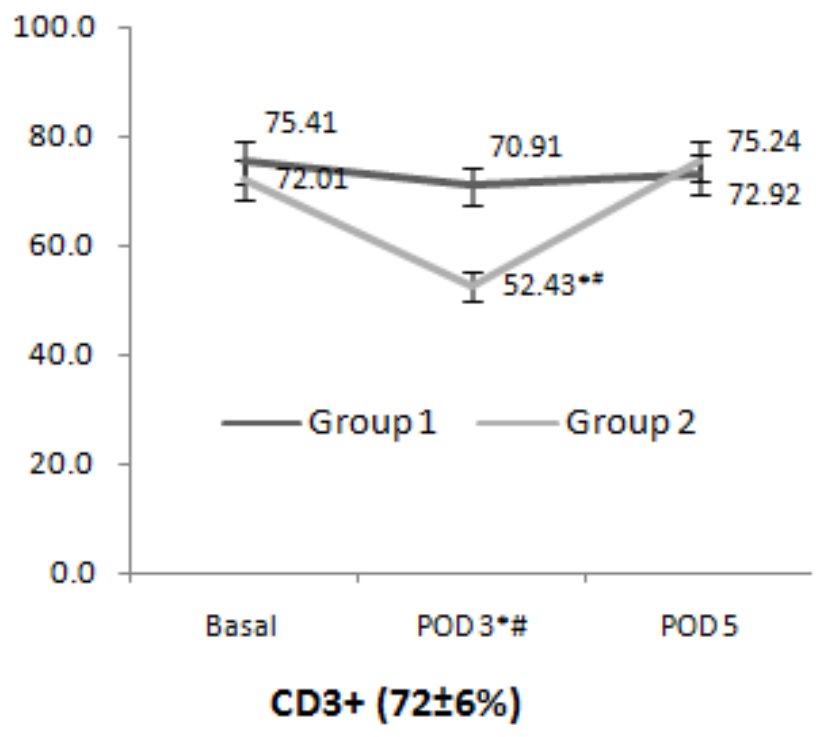

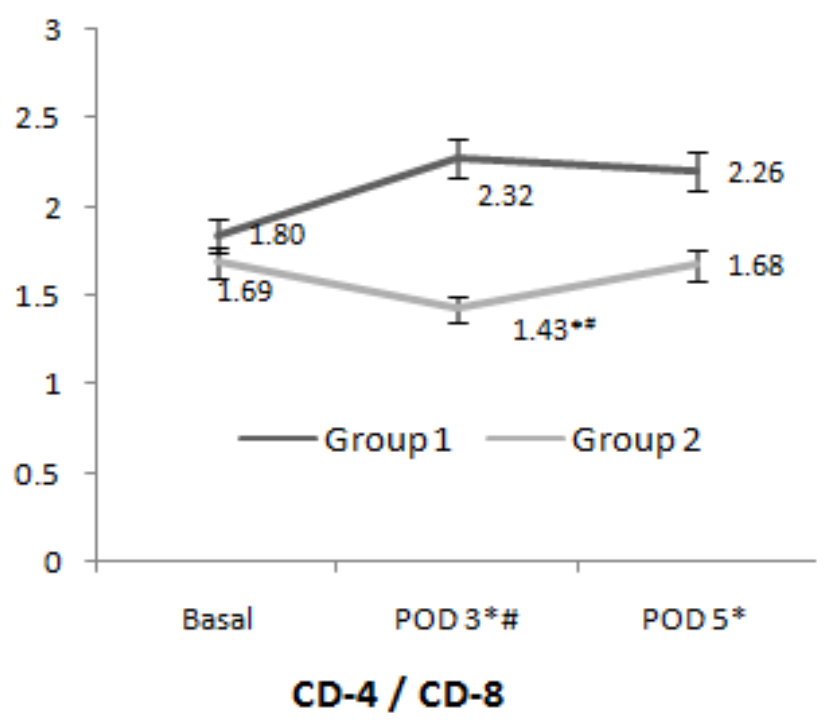

Figure 2: Assessment of the CD-3 and CD4/CD8 Ratio at Points 1, $6^{* \#}$ and $7^{*}(* P<0.05$ for comparison between groups, $\# P<0.05$ for comparison with the baseline).

\section{Cognitive function}

The assessment of the cognitive function has indicated a lower total score in the postoperative period as compared with the baseline in both groups. However, this decrease was only significant in Group 2 patients and on POD 1 (Figure 3). At the same time, patients in both groups had completely restored their total test scores according to all the scales and questionnaires by POD 5, which did not worsen until their discharge. We have shown that the initial cognitive functionality in both groups lied within the normal values, along with the other parameters of the 
biochemical and immunological tests conducted.

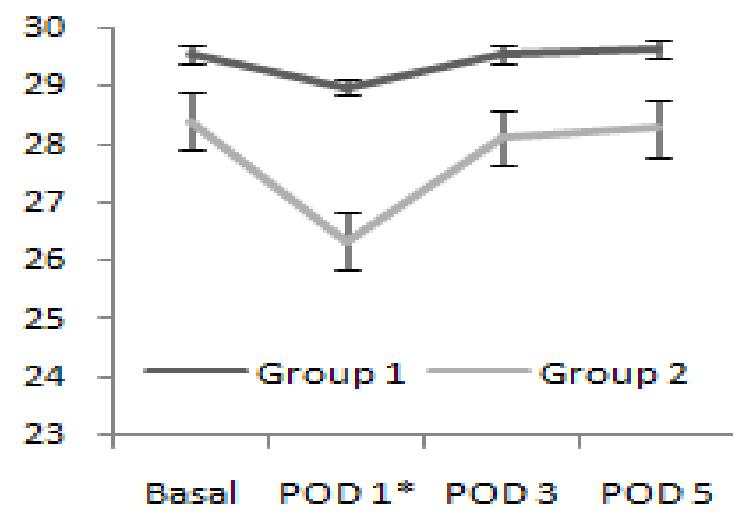

MMSE

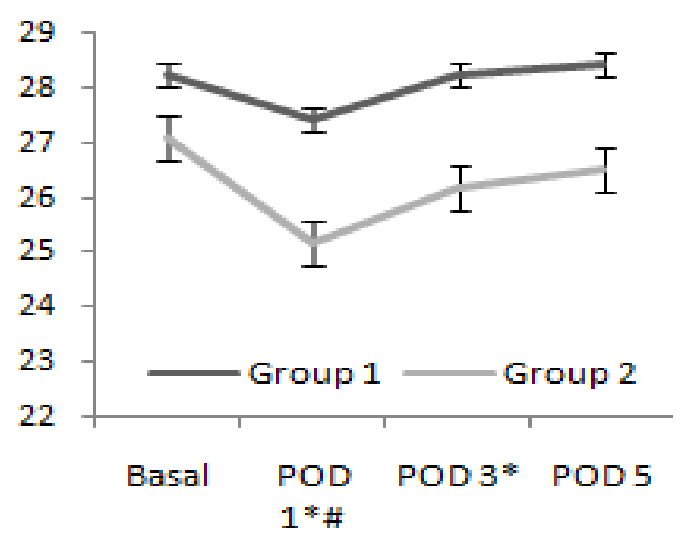

MoCA

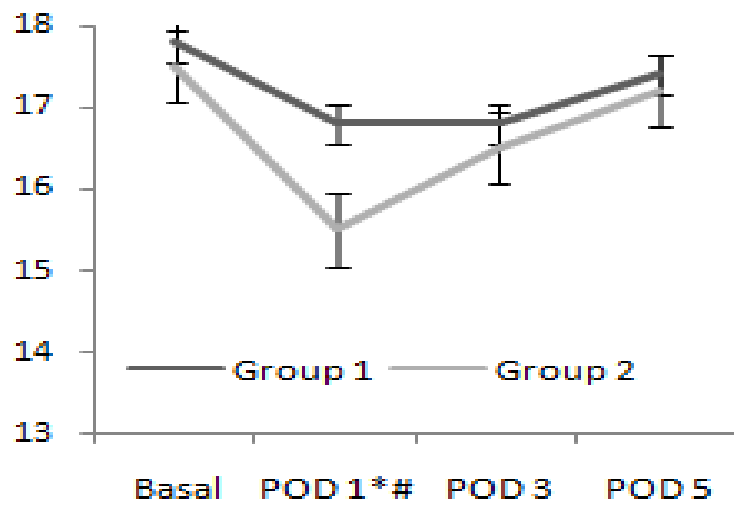

FAB

Figure 3: Assessment of the cognitive functions at Points 1, 5, 6, and 7 $\left({ }^{*} P<0.05\right.$ for comparison between groups, $\# P<0.05$ for comparison with the baseline).

For the whole period of the study, no case of postoperative delirium has been observed in both groups, and not a single instance of the development of a severe cognitive dysfunction has been detected in the postoperative period. The total scores of the cognitive tests have indicated a strong negative correlation with the levels of peripheral inflammatory markers on POD 1. The MMSE test scores in Group 2 on POD 1 have indicated a moderate negative correlation with the levels of IL-6 (Spearman rank correlation $\mathrm{R}=-0.4, p=0.0011,0.95 \mathrm{CI}$ ).
A comparative assessment of the FAB test results has revealed no significant deviations in the initial scores in both groups. However, Group 2 has shown a significant drop in the points scored on POD 1 (Figure 3). It should be noted that the most severe weakening of the frontal function has manifested itself in decreased dynamic praxis. Patients in both groups took a longer time to complete the test On POD 1 and POD 3, the number of mistakes and unfulfilled assignments going up.

It is noteworthy that the results of all the three tests (MMSE, MoCA, and FAB) in both groups tend to approach the preoperative levels on POD 5 and POD 7, with no significant differences between the groups having been found.

\section{Discussion}

The study has demonstrated that inadequate pain relief after surgery can result in a wide range of metabolic disorders, which may adversely affect the recovery from spinal surgery. Pain directly stimulates sympathetic activity, which manifests itself in the increased plasma levels of adrenaline and noradrenalin, and also stimulates the metabolic disorders of glucose and cortisol, and immune dysfunction. We can conclude that epidural analgesia positively affects the immune system, preventing severe postoperative immuno suppression, which is particularly due to the increase in the number of CD-3-cells, B-cells, CD4/ CD8 ratio, and partially of NK-cells. In a previous study, higher cortisol levels postoperatively were shown to be associated with a higher chance of developing POCD ${ }^{[4,5,7,8]}$.

Epidural anesthesia has an important influence on the modulation of the immune response in patients who underwent major orthopedic surgery. This study has shown that combined general and epidural anesthesia during and after reconstructive surgery on the spine provides better pain relief and hence early patients' mobilization.

Rasmussen et al. ${ }^{[16]}$ demonstrated no significant distinction between general and regional anesthesia in rates of POCD; however, another study showed a significantly poorer cognitive function in patients who had been administered general rather than regional anesthesia ${ }^{[17]}$. Each study included patients getting noncardiac surgery, but both had biases such as large dropout rates and failure to account for social factors. As previously discussed, regional anesthesia leads to an attenuated stress response in comparison to general anesthesia. This suggests that although levels of surgical stress can be associated with POCD, its role as a causative factor is still uncertain. In our research, we have completely confirmed the previous data regarding the stress response modulation and attenuating the immune suppression.

Our study has demonstrated that the total test scores in the MoCA and MMSE tests were insignificantly reduced in Group 1. At the same time, Group 2 patients have revealed more serious cognitive impairments, but of a transient and mild character (Figure 3). Moderate negative correlations of total scores in the MoCA and MMSE tests have also been found with the level of C-reactive protein, and the need for opioids on the surgery day and on POD 1.

According to the results of cognitive assessment, patients of the age of about sixty have displayed moderate disturbances of dynamic praxis, which was manifested in difficulties comprehending the action program, memorizing facts and in impaired speech productivity. Such decline in cognitive func- 
tionality was a natural consequence of inactivity and exhaustion on POD 1.

In Group 2, eight patients (32\%) showed moderate cognitive dysfunction on POD 1, normalizing towards POD 5; the application of Fisher test has demonstrated significant differences between groups (five patients or $16 \%$ in Group 1, $P<0$, $05)$.

The present study has indicated that surgical stress response in middle-aged patients after orthopedic spinal surgery manifests itself in a significant increase of inflammatory markers, particularly IL-6, IL-1, IL-10, C-reactive protein, and in immune dysfunction. At the same time, the severity of the post-operative cognitive decline, estimated with the aid of standard scales and questionnaires, showed strong correlation with the level of inflammation markers on POD 1, particularly with the level of proinflammatory interleukin-6. The levels of IL-6 have also strong or moderate positive correlations with cortisol, C-reactive protein, and the severity of self-rated pain.

Nevertheless, Group 1 patients have demonstrated the absence of any significant cognitive deficit in the early period after spinal surgery, as compared with the baseline level of their cognitive functionality. Group 2 patients have displayed a statistically significant reduction in their neuropsychological testing scores, as compared with Group 1 and also with the preoperative data. The most considerable changes have been associated with the impairment of short-term memory, speech fluency and productivity, and the power of concentration. However, these symptoms were not severe or fatal, and corresponded to the moderate and short-term cognitive dysfunction in the early postoperative period.

A study by van Harten et al. shows that immunological reaction is one of the most important causative factors of cardiac POCD $^{[21]}$. Buvanendran also finds that cardiac and non-cardiac POCD are associated with inflammatory reaction ${ }^{[22]}$. The researchers have shown that IL-6 and S-100 $\beta$ are the POCD-related pro-inflammatory markers; their meta-analysis strengthens the clinical evidence that POCD is accompanied by inflammatory reaction. However, other proinflammatory cytokines, such as IL-10 and IL-8, were not involved in this analysis due to the limited number of studies available.

In the present study, we have conducted a comparative clinical analysis of two homogeneous groups falling within the age range of about sixty and featuring a minimum number of risk factors for the POCD development (surgical intervention not more than three lumbar segments, its duration under three hours, the blood loss under $500 \mathrm{ml}$ ). The groups had comparable limits of the BIS-index and systemic hemodynamics.

In the present-day neurosurgery and anesthesiology, both anesthetic techniques in question are believed to be adequate and acceptable for spinal surgery. However, we still cannot make a conclusion that epidural anesthesia has immediate impact on the POCD development, due to insufficient data and the absence of the follow-up period. We can only suggest that the measures restricting surgical stress response and immune suppression (e.g. epidural blockades) indirectly minimize the risk of POCD in middle-aged patients in the early postoperative period after spinal surgery.

\section{Conclusion}

Epidural anesthesia in spinal surgery has been a major factor limiting the surgical stress response. EA has proved to be the most adequate analgesia and yielded the highest total scores in the cognitive tests on POD 1, as compared with general sevoflurane anesthesia and postoperative opioid analgesia. Furthermore, EA can modulate adaptive immunity by improving the distribution of white blood cells.

Due to little clinical material, lack of follow-up and insufficient evidence of the immediate effect of epidural anesthesia on POCD, further research is required to obtain more results regarding the pathogenesis of postoperative cognitive alterations after spinal surgery in middle-aged and elderly patients, and to explicate the relationship between cognitive functionality and inflammatory/immune changes, including processes in the central nervous system.

Conflict of interest: The authors declare no conflict of interest. 


\section{References}

1. Zhang, M.D., Barde, S., Yang, T., et al. Orthopedic surgery modulates neuropeptides and BDNF expression at the spinal and hippocampal levels. (2016) Proc Natl Acad Sci U S A 113(43): E6686-E6695.

Pubmed | Crossref | Others

2. Choi, S., Rampersaud, Y.R., Chan, V.W., et al. The addition of epidural local anesthetic to systemic multimodal analgesia following lumbar spinal fusion: a randomized controlled trial. (2014) Can J Anaesth 61(4): 330-339.

Pubmed | Crossref | Others

3. Hirsch, J., Vacas, S., Terrando, N., et al. Perioperative cerebrospinal fluid and plasma inflammatory markers after orthopedic surgery. (2016) J Neuroinflammation 13(1): 2-11.

Pubmed | Crossref | Others

4. Iwasaki, M., Edmondson, M., Sakamoto, A., et al. Anesthesia, surgical stress, and "long-term" outcomes. (2015) Acta Anaesthesiol Taiwan 53(3):99-104

Pubmed | Crossref | Others

5. Yang, Y., Zhao, X., Dong, T., et al. Risk factors for postoperative delirium following hip fracture repair in elderly patients: a systematic review and meta-analysis. (2017) Aging Clin Exp Res 29(2): 15-126.

Pubmed | Crossref | Others

6. Steinmetz, J., Christensen, K.B., Lund, T., et al. Long-term consequences of postoperative cognitive dysfunction. (2009) Anesthesiology 110(3): 548-555.

Pubmed | Crossref | Others

7. Vacas, S., Degos, V., Feng, X., et al. The neuroinflammatory response of postoperative cognitive decline. (2013) Br Med Bull 106: 161-178. Pubmed | Crossref | Others

8. Passos, I.C., Vasconcelos-Moreno, M.P., Costa, L.G., et al. Inflammatory markers in post-traumatic stress disorder: a systematic review, meta-analysis, and meta-regression. (2015) Lancet Psychiatry 2(11): 1002-1012.

Pubmed | Crossref | Others

9. Berger, M., Nadler, J.W., Browndyke, J., et al. Postoperative cognitive dysfunction: minding the gaps in our knowledge of a common postoperative complication in the elderly. (2015) Anesthesiol Clin 33(3): 517-550.

Pubmed | Crossref | Others

10. Riedel, B., Browne, K., Silbert, B. Cerebral protection: inflammation, endothelial dysfunction, and postoperative cognitive dysfunction. (2014) Curr Opin Anaesthesiol 27(1): 89-97.

Pubmed | Crossref | Others

11. Kazmierski, J., Banys, A., Latek, J., et al. Cortisol levels and neuropsychiatric diagnosis as markers of postoperative delirium: a prospective cohort study. (2013) Crit Care 17(2): R38.

Pubmed | Crossref | Others
12. Peng, L., Xu, L., Ouyang, W. Role of peripheral inflammatory markers in postoperative cognitive dysfunction (POCD): a meta-analysis. (2013) PLoS One 8(11): e79624.

Pubmed | Crossref | Others

13. Rizk, P., Morris, W., Oladeji, P., Huo M. Review of postoperative delirium in geriatric patients undergoing hip surgery. (2016) Geriatr Orthop Surg Rehabil 7(2): 100-105.

Pubmed | Crossref | Others

14. Li, Y.C., Xi, C.H., An, Y.F., et al. Perioperative inflammatory response and protein S-100 $\beta$ concentrations - relationship with post-operative cognitive dysfunction in elderly patients. (2012) Acta Anaesthesiol Scand 56(5): 595-600

Pubmed | Crossref $\mid$ Others

15. Terrando, N., Monaco, C., Ma, D., et al. Tumor necrosis factor-alpha triggers a cytokine cascade yielding postoperative cognitive decline. (2010) Proc Natl Acad Sci U S A 107(47): 20518-20522.

Pubmed | Crossref | Others

16. Rasmussen, L.S., Christiansen, M., Rasmussen, H. Do blood concentrations of neuron specific enolase and S-100 beta protein reflect cognitive dysfunction after abdominal surgery? ISPOCD Group. (2000) Br J Anaesth 84(2): 242-244.

Pubmed $\mid$ Crossref $\mid$ Others

17. Mandal, S., Basu, M., Kirtania, J., et al. Impact of general versus epidural anesthesia on early post-operative cognitive dysfunction following hip and knee surgery. (2011) J Emerg Trauma Shock 4(1): 23-28.

Pubmed | Crossref | Others

18. Zywiel, M.G., Prabhu, A., Perruccio, A.V., et al. The influence of anesthesia and pain management on cognitive dysfunction after joint arthroplasty: a systematic review. (2014) Clin Orthop Relat Res 472(5): 1453-1466.

Pubmed | Crossref | Others

19. Oh, E.S., Li, M., Fafowora, T.M., et al. Preoperative risk factors for postoperative delirium following hip fracture repair: a systematic review. (2015) Int J Geriatr Psychiatry 30(9): 900-910.

Pubmed $\mid$ Crossref $\mid$ Others

20. Jiang, X., Chen, D., Lou, Y., et al. Risk factors for postoperative delirium after spine surgery in middle- and old-aged patients. (2016) Aging Clin Exp Res.

Pubmed | Crossref | Others

21. Van Harten, A.E., Scheeren, T.W.L., Absalom, A.R. A review of postoperative cognitive dysfunction and neuroinflammation associated with cardiac surgery and anaesthesia. (2012) Anaesthesia 67(3): 280-293.

Pubmed | Crossref | Others

22. Buvanendran, A., Kroin, J.S., Berger, R.A., et al. Upregulation of prostaglandin E2 and interleukins in the central nervous system and peripheral tissue during and after surgery in humans. (2006) Anesthesiology 104(3): 403-410.

Pubmed $\mid$ Crossref $\mid$ Others
Ommega Online Publishers

Journal Title: Journal of Anesthesia and Surgery (JAS)

Journal Short Name: J Anesth Surg
Journal ISSN: 2377-1364

E-mail: anestheisa@ommegaonline.com

Website: www.ommegaonline.org 